\title{
Outcome of Surgically Managed Diaphyseal Fractures in Children- A Prospective Study
}

\author{
Sridhar Maryada Reddy ${ }^{1}$, Nareshkumar Satyanarayan Dhaniwala ${ }^{2}$ \\ ${ }^{1}$ Department of Orthopaedics, JNMC, DMIMS, Sawangi, Wardha, Maharashtra, India. \\ ${ }^{2}$ Department of Orthopaedics, JNMC, DMIMS, Sawangi, Wardha, Maharashtra, India.
}

\section{ABSTRACT}

\section{BACKGROUND}

Childhood injuries have become a global public health concern. It is estimated that $10-25 \%$ of paediatric injuries are fractures. The definitive treatment of paediatric diaphyseal fractures has always remained controversial and determined by surgical experience and local trends of practice. The aim of this study was to study the outcome of surgically managed diaphyseal fractures in children.

\section{METHODS}

A prospective interventional study was done comprising of 42 fractures in 28 children having displaced diaphyseal fractures of major long bones. Flynn's scoring criteria was used to study the outcome.

\section{RESULTS}

The observations of this study are based on 42 surgically managed diaphyseal fractures in long bones in 28 children. The mean age of patients was 10.85 years. The commonest mode of injury was fall while playing (from level ground) (18 cases, $4.29 \%$ ). The commonest bones to get fractured were both radius and ulna together (50\%), followed by femur (25\%), tibia $(21.43 \%)$ and ulna singly $(3.57 \%)$. The commonest location of fracture was middle third shaft (25 fractures, 59.52\%). Transverse fractures accounted for major proportion of this series. Among the 42 fractures, 39 fractures were treated with titanium elastic nailing and 2 compound fractures were treated with debridement and external fixator application. One 15 year old girl was treated by femur interlock nailing. Among the 25 children who could be followed up, the average follow up period was 22.08 weeks. The mean period of union was 10.69 weeks. Majority of the fractures had excellent outcome (30 fractures, $76.92 \%$ ), 7 fractures $(17.95 \%$ ) had satisfactory outcome while only 2 fractures $(5.13 \%)$ had poor outcome.

\section{CONCLUSIONS}

Titanium elastic nailing (TENS) is found to be an effective method of treating closed displaced diaphyseal fractures in children. It is better than plaster cast immobilization due to shorter period required for union, better fracture reduction and stabilization besides prevention of stiffness of adjacent joints and fracture disease. More use of this method is recommended due to these advantages.
Corresponding Author: Dr. Sridhar Maryada Reddy, Department of Orthopaedics, JNMC, DMIMS, Sawangi, Wardha, Maharashtra, India. E-mail: sridharmreddy90@gmail.com

DOI: $10.14260 / j e m d s / 2020 / 90$

Financial or Other Competing Interests: None.

How to Cite This Article:

Reddy SM, Dhaniwala NS. Outcome of surgically managed diaphyseal fractures in children- a prospective study. J. Evolution Med. Dent. Sci. 2020;9(07):393-397, DOI: 10.14260/jemds/2020/90

Submission 10-12-2019, Peer Review 22-01-2020,

Acceptance 28-01-2020,

Published 17-02-2020.

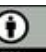

\section{KEY WORDS}

Paediatric, Fractures, Diaphyseal, TENS. 


\section{BACKGROUND}

Childhood injuries have become a global public health concern. As per World Health Organization (WHO), it is estimated that over $6,30,000$ children under the age of 15 years were killed by an injury. It is also estimated that for every child who dies, several thousands of children have nonfatal injuries and live on with varying degrees of disability ${ }^{1}$. It is estimated that $10-25 \%$ of paediatric injuries are fractures. ${ }^{2}$ Fractures are found to be more common in boys. Boys have a lifetime risk of sustaining a fracture as high as $64 \%$ while for girls, it is $40 \%$. Falls (27\%) are the leading cause of fractures in children, among which the majority of the fractures are a result of fall from a height less than 1 meter, followed by fall from unknown height and then by fall from height more than 1 meter.

Accidents occurring during leisure activities (25\%) also formed a major cause of fractures in children. Fractures have a considerable impact on daily living and activities of affected children and thus are an important topic of public health. ${ }^{3}$ Fractures sustained in children mostly heal without loss of function, but contribute to significant associated costs for the child and family, including time away from school and reduced activities for a considerable time. ${ }^{4}$ The definitive treatment of paediatric diaphyseal fractures has always remained controversial. The management varies with age, site of fracture and fracture pattern. It is also determined by surgical experience and local trends of practice. For preschool children, non-surgical treatment continues to be the treatment of choice. However, unlike pre-school children, patients in the intermediate age group of 5-15 years have a higher risk of malunion and shortening with early closed reduction and cast application. Use of traction for a couple of weeks before cast application can maintain length until early healing has occurred.

As a result, older children who are managed with traction and cast, may end up missing several months of school until full union has been achieved. Today, the trends have veered towards operative fixation due to the benefits of early mobilization and shorter hospital stays.5,6

Ideally, fixation of paediatric diaphyseal fractures should be able to produce an internal splint that maintains reduction till callus is formed, shares loads and does not endanger the physis or vascularity. Several studies have shown that titanium elastic nailing system/ flexible intramedullary nailing fixation meets these requirements. It allows rapid mobilization, has potentially no risk for osteonecrosis, low risk for physeal injury and reduced risk for refracture. ${ }^{7}$ Surgery is an effective method of treating closed diaphyseal fractures in paediatric age group.

It is better than plaster cast immobilization due to shorter period required for union, better fracture reduction and stabilization besides prevention of stiffness of adjacent joints and fracture disease. The various types of implants available include Titanium elastic nails (TENS), Rush nails, solid intramedullary nails, external fixators, etc. The aim of this study was to evaluate the outcome of surgically managed diaphyseal fractures in paediatric age group.

\section{METHODS}

This was a prospective interventional study done in the Department of Orthopaedics in AVBRH, Sawangi, Wardha from May 2017 to September 2019. Institutional Ethical committee (IEC) clearance was obtained. All the patients in the age group of 3-15 years visiting the outpatient department or the emergency department, having displaced diaphyseal fracture in a major long bone (humerus/ radius/ ulna/ femur/ tibia) and willing to undergo surgical management were included in the study, after obtaining a written informed consent from parents/guardians. Pathological fractures, fractures close to the proximal and distal epiphyseal plate and patients unfit for surgery were excluded from the study. Total 28 children with 42 fractures were included and the treatment was decided by the type of fracture and its pattern. Treatment included either closed or open reduction or internal fixation with Titanium elastic nails (TENS) under fluoroscopy. In case of compound fractures, debridement of the wound was done, followed by external fixator application. Patients were followed up at 3 weeks, 6 weeks, 12 weeks, 24 weeks and 9 months. At each follow up, physical examination and radiological examination of operated limb was done. Final outcome was graded as excellent or satisfactory or poor as per Flynn's scoring criteria. ${ }^{7}$ Flynn's scoring criteria is based on limb length inequality, malalignment, pain and complication.

\section{Statistical Analysis}

Statistical analysis was done by using descriptive and inferential statistics using statistical tests of significance. The software used in the analysis was SPSS 22.0 version and Graph Pad Prism 6.0 version and $\mathrm{p}<0.05$ was considered as level of significance.

\section{RESULTS}

The observations of this study are based on 42 surgically managed diaphyseal fractures in long bones in 28 children. Among the 28 children, 1 child expired due to an unrelated medical cause while 2 were lost to follow up. The mean age of patients in the study was 10.85 years (range 3-15 years). $60.71 \%$ were boys while $39.29 \%$ were girls.

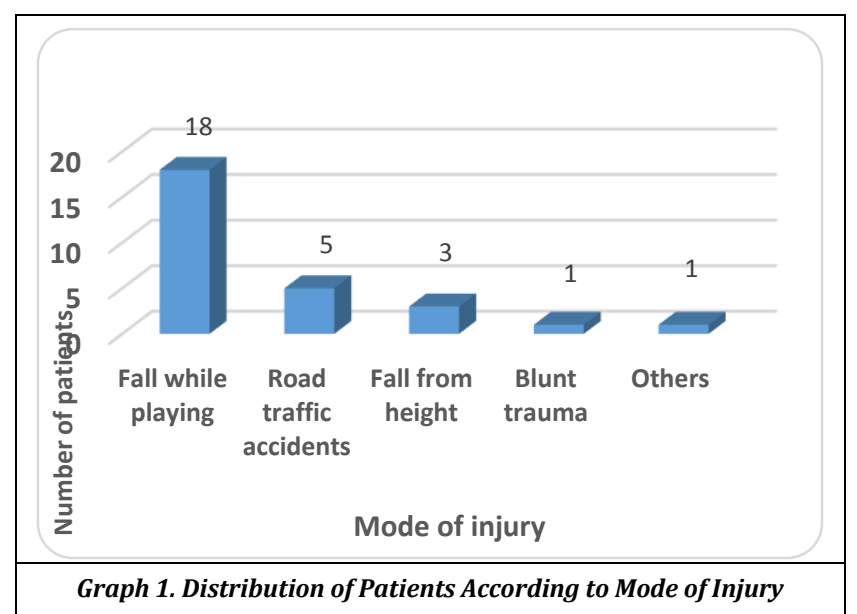


The commonest mode of injury was fall while playing (graph 1). One child, a known case of seizure disorder, sustained fracture as a result of fall while convulsing (3.57\%). The commonest bones to get fractured were both radius and ulna together (50\%), followed by femur (25\%), tibia $(21.43 \%)$ and ulna alone (3.57\%). The commonest location of fracture was middle third shaft (25 fractures, 59.52\%). Majority of the fractures were seen on the left side $(26$ fractures, $61.9 \%)$ whereas 16 fractures $(38.1 \%)$ were seen on the right side. Transverse fractures accounted for major proportion of this series (32 cases, 76.19\%) followed by oblique fractures (06 cases, $14.29 \%$ ). Out of the 42 fractures, 3 were compound while rest all were closed. Among open fractures, 2 were of Gustilo Anderson type 1 while 1 was Gustilo Anderson type 3B. Among the 42 fractures, 39 fractures were treated with Titanium elastic nailing. One girl having shaft femur fracture was treated with femur interlock nail, as her growth plate was fused. 2 compound fractures were treated with debridement and external fixator application. Among these 2, in one case, external fixator was removed after 3 weeks when the wound healed and was then managed with titanium elastic nailing. In the other case, external fixator was continued till 6 weeks after which it was removed as signs of union were seen.

Total follow up period ranged from 9 weeks to 39 weeks. 4 patients completed the 9 months ( 39 weeks) follow up, 12 patients completed the 24 weeks follow up and 9 patients completed the 12 weeks follow up. Among these 25 cases who came for follow up, the average follow up period was 22.08 weeks. Results were assessed at the last follow up of the patient.

The mean period of union was 10.69 weeks, the range being between 6 weeks to 39 weeks. For 37 cases which were primarily managed with titanium elastic nailing, the mean period of union was 9.57 weeks, whereas the 2 compound fractures united with a mean period of 31.5 weeks. Entry site skin irritation was found to be the most common complication. Two cases had delayed union. Out of 39 fractures, 8 fractures (20.51 \%) showed angulatory deformity. Majority of cases had $<5^{0}$ angulatory deformity ( 5 cases, $12.82 \%$ ) which is expected to get corrected by remodelling. Lengthening and shortening were observed in 2 cases each. The majority of the fractures had excellent outcome (30 fractures, 76.92\%), 7 fractures (17.95\%) had satisfactory outcome while only 2 fractures $(5.13 \%)$ had poor outcome.

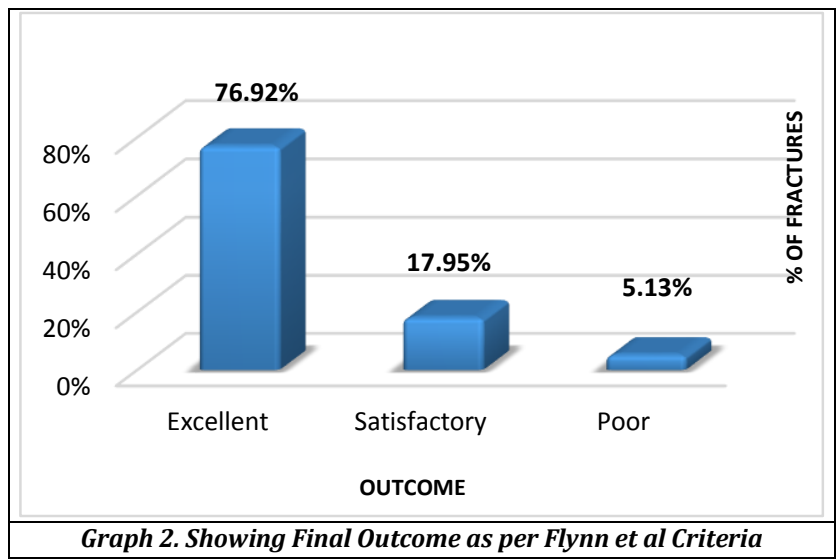

\section{DISCUSSION}

In the past, paediatric long bone fractures were treated by conservative means like traction, hip spica application, cast application etc. To avoid the effects of prolonged immobilization, to reduce the loss of school days and for better nursing care, the operative approach has been gaining popularity for last two decades. ${ }^{8}$ Though there are a variety of options available for surgically managing diaphyseal fractures in children, there has been a growing trend towards titanium elastic nails fixation. Encouraging results with its studies have increased the use of titanium elastic nails in the management of diaphyseal fractures in children. In paediatric fractures, the periosteum is not disturbed. Fixing with titanium elastic nails is mostly a closed procedure. Hence, there is no disturbance of fracture hematoma, thereby less risk of infection. This technique can be adapted to treat almost any long bone fracture in children. In contrast with techniques involving rigid fixation, stability is not only ensured by the nails but also by the bone and surrounding soft tissue. The nails provide internal elastic support, channelling forces and prevent excessive displacement by the automatic adjustments of the bone fragments. The bone provides axial stability provided there is no overlap at fracture site. This is ensured either by cortical contact in end to end reduction or by anchoring the nails in the metaphysis. ${ }^{9}$

The mean age in our study was 10.85 years. Youngest child was 3 years old and eldest child was 15 years old. In the study by Baig MS et al,10 majority of the patients (46.7\%) were in the age group 9-12 years, followed by 13-16 years age group (33.3\%), with the average age being 10.93 years. Kemal K et al. ${ }^{11}$ in their study comprising of children between 6-15 years, found the mean age to be 8.6 years. Flynn JM et al. ${ }^{7}$ had a mean age of 9.5 years while Arora R et al. ${ }^{12}$ had a mean age of 8.62 years in their respective studies. In the present study, there were 17 boys (60.71\%) and 11 girls (39.29\%). Similar preponderance of males was found in studies conducted by Ghilley SK et al. ${ }^{13}$ (64\% boys and 36\% girls), Baig MS et al. ${ }^{10}$ (73.3\% boys and $26.7 \%$ girls), Tandon T et al. ${ }^{14}$ (54.8\% boys and $45.2 \%$ girls), Babu A et al. ${ }^{15}$ (78.6 $\%$ were males and $21.3 \%$ were females) and Arora R et al. ${ }^{12}$ (67\% males, 33\% females). Male preponderance in all these studies can be attributed to higher level of activity among male children as compared to female children.

We found fall from level ground while playing (64.29\%) to be the most common mode of trauma, followed by road traffic accident $(17.86 \%)$. Other causes were fall from height $(10.71 \%)$, blunt trauma $(3.57 \%)$ and seizures $(3.57 \%)$. In a study by Singh 0 et al, $1659.40 \%$ patients had history of fall, followed by road traffic accident (32\%). Baig MS et al. ${ }^{10}$ and Babu A et al. ${ }^{15}$ found the predominant cause to be road traffic accidents. Many other studies also found road traffic accidents to be the predominant cause. The reasons for this difference may be attributed to the fact that our study was based in a rural scenario, where use of motorized vehicles is less compared to cities. In our study, fracture of radius and ulna together was the commonest ( $50 \%)$, followed by femur (25\%), tibia $(21.43 \%)$ and ulna alone $(3.57 \%)$. Cheng JC et al. ${ }^{17}$ and Tandon $\mathrm{T}$ et al. ${ }^{14}$ found the most common fracture site was distal radius. Baig MS et al. ${ }^{10}$ conducted a study on diaphyseal fractures in which the most common fractures 
were femoral fractures (56.7\%). The varying values of bone involved in fracture could be because of difference in population, selection methods and rural/ urban setting.

The most common level of fracture was middle third shaft $(59.52 \%)$, followed by proximal third shaft $(21.43 \%)$ and distal third shaft (19.05\%). Arora R et al,12 Zionts LE et al. ${ }^{18}$ and Flynn JM et al. ${ }^{7}$ also found the most common level to be middle third shaft. In our study, left sided fractures were more common (61.9\%) than right sided injuries $(28.1 \%)$. Similar findings were noted by Zionts LE et al. ${ }^{18}(72 \%$ left and $28 \%$ right sided) and Teoh $\mathrm{KH}$ et al. ${ }^{19}$ (59 \% left, 41\% right sided), whereas study by Vaish A et al. ${ }^{20}$ (71\% right, $29 \%$ left) showed more right sided fractures. The most common fracture pattern was transverse $(76.19 \%)$, followed by oblique (14.29\%). Gilley SK et al. ${ }^{13}$ too found transverse fractures (44\%) to be the most common in his study, followed by oblique fractures.

The mean period of union among the 39 fractures was 10.69 weeks. The mean period of union among the 37 fractures which were primarily managed with titanium elastic nails was 9.57 weeks, whereas for 2 cases which were primarily managed with external fixator application, the mean period of union was 31.5 weeks. In a study by Kubaik EN et al. ${ }^{21}$ in which they studied fractures of tibia treated with titanium elastic nails and external fixators, they found the mean period of union was 7 weeks for cases treated with TENS while for external fixator cases, the mean period of union was 18 weeks. The observations show that union occurs faster with internal fixation with TENS, while open fracture take more time due to loss of fracture hematoma, despite adequate stabilization with external fixator.

Nails caused entry site irritation at $6(15.38 \%)$ entry sites. In the study done by Flynn JM et al. ${ }^{7} 4$ patients (7\%) out of 58 had entry site irritation due to prominent nail tip. Entry site irritation is the most common complication of TENS. ${ }^{7} \mathrm{New}$ instrumentation, allowing nail removal when only a few millimeters of nail is outside, may solve this problem. ${ }^{7}$ There was shortening seen in 2 cases (8\%) and lengthening seen in 2 cases (8\%). Flynn JM et al. ${ }^{7}$ noted limb length discrepancy in 6 cases $(10.3 \%)$. In the study by Ghilley SK et al. ${ }^{13} 22 \%$ of cases showed limb length discrepancy. Ligier JN et al. ${ }^{9}$ found 4 cases of lengthening more than $1 \mathrm{~cm}$ and 3 cases of shortening more than $1 \mathrm{~cm}$. Our study results are comparable to the above studies. There was malalignment of 15 degrees in 1 case $(2.56 \%), 7$ degrees in 1 case $(2.56 \%)$ and 6 degrees in 1 case $(2.56 \%)$. Malalignment up to 5 degrees was observed in 5 cases $(12.82 \%)$. In the study done by JM Flynn et al. 7 , there were $6(10.3 \%)$ cases of angular deformity $>5$ degrees while Ghilley SK et al. ${ }^{13}$ found 3 cases (9.35\%) of angular deformity $>5$ degrees. To avoid any residual angulation, it is important that the nails are introduced at the same level and have identical curvatures. ${ }^{7}$

In the present study, the result was excellent in 30 fractures $(76.92 \%)$, satisfactory in 7 fractures $(17.95 \%)$ and poor in 2 fractures (5.13\%). Poor result was observed in both the fractures due to delayed union, shortening and angulation. In the study by J M Flynn et al. ${ }^{7}$ there were $65.5 \%$ excellent results, $31.03 \%$ satisfactory and $1.72 \%$ poor result. In the study by Vaish A et al.20 they obtained excellent outcome in $73 \%$, satisfactory outcome in $27 \%$ cases, based on Flynn's scoring criteria. Ghilley SK et al. ${ }^{13}$ found $80 \%$ cases had excellent outcome while $20 \%$ had satisfactory outcome. Kumar P et al. ${ }^{22}$ found $75 \%$ excellent, $15.6 \%$ satisfactory and $9.4 \%$ poor outcome.

\begin{tabular}{|c|c|c|c|}
\hline \multirow{2}{*}{ Studies } & \multicolumn{3}{|c|}{ Results } \\
\cline { 2 - 4 } & Excellent & Satisfactory & Poor \\
\hline J M Flynn et al. ${ }^{11}$ & $65.5 \%$ & $31.03 \%$ & $1.72 \%$ \\
\hline Vaish A et al. $^{76}$ & $73 \%$ & $27 \%$ & - \\
\hline Ghilley SK et al. $^{79}$ & $80 \%$ & $20 \%$ & - \\
\hline Kumar P et al. ${ }^{8}$ & $75 \%$ & $15.6 \%$ & $9.4 \%$ \\
\hline Present Study & $76.92 \%$ & $17.95 \%$ & $5.13 \%$ \\
\hline Outcome in Various Studies Compared to the Present Study \\
\hline
\end{tabular}

\section{CONCLUSIONS}

Titanium elastic nailing is found to be an effective method of treating closed displaced diaphyseal fractures in children. It is better than plaster cast immobilization due to shorter period required for union, better fracture reduction and stabilization besides prevention of stiffness of adjacent joints and fracture disease. More use of this method is recommended due to these advantages. Fixation with titanium elastic nails offers better modality of treatment in long bones of children and should be used more often whenever indicated.

\section{REFERENCES}

[1] Child injuries. World Health Organization, 2014 [cited $2018 \quad$ Oct 1]. https://www.who.int/violence_injury_prevention/chil d/injury/en/

[2] Landin LA. Epidemiology of children's fractures. Journal of Pediatric Orthopedics Part B 1997;6(2):79-83.

[3] Joeris A, Lutz N, Wicki B, et al. An epidemiological evaluation of pediatric long bone fractures-a retrospective cohort study of 2716 patients from two Swiss tertiary pediatric hospitals. BMC Pediatrics 2014;14(1):314.

[4] Randsborg PH, Gulbrandsen P, Benth JŠ, et al. Fractures in children: epidemiology and activity-specific fracture rates. JBJS 2013;95(7):e42.

[5] Khoriati AA, Jones C, Gelfer Y, et al. The management of paediatric diaphyseal femoral fractures: a modern approach. Strategies in Trauma and Limb Reconstruction 2016;11(2):87-97.

[6] Sela Y, Hershkovich O, Sher-Lurie N, et al. Pediatric femoral shaft fractures: treatment strategies according to age --13 years of experience in one medical center. Journal of Orthopaedic Surgery and Research 2013;8(1):23.

[7] Flynn JM, Hresko T, Reynolds RA, et al. Titanium elastic nails for pediatric femur fractures: a multicenter study of early results with analysis of complications. Journal of Pediatric Orthopaedics 2001;21(1):4-8.

[8] Conway B. The effect of hospitalization on adolescence. Adolescence 1971;6:77-92.

[9] Ligier JN, Metaizeau JP, Prévot J, et al. Elastic stable intramedullary nailing of femoral shaft fractures in children. The Journal of Bone and Joint Surgery British 1988;70(1):74-7. 
[10] Baig MS, Thutari N, Kodandapani K, et al. Comprehensive study of management of diaphyseal fractures of long bones in children by titanium elastic nailing system. Journal of Evolution of Medical and Dental Sciences 2019;8(13):969-74.

[11] Kayaokay K, Aktuglu K. Titanium elastic nailing in pediatric femoral diaphyseal fractures in the age group of 6-15 years mid-term and long-term outcomes. Pakistan Journal of Medical Sciences 2018;34(6):152933.

[12] Arora R, Mishra P, Aggarwal AN, et al. Factors responsible for redisplacement of pediatric forearm fractures treated by closed reduction and cast: Role of casting indices and three point index. Indian Journal of Orthopaedics 2018;52(5):536-47.

[13] Ghilley SK, Meena MK, Jhanwar P, et al. Use of flexible intramedullary nailing in treating diaphyseal fractures of long bone of lower limb in children. Journal of Orthopedics, Traumatology and Rehabilitation 2019;11(1):21-6.

[14] Tandon T, Shaik M, Modi N. Paediatric trauma epidemiology in an urban scenario in India. Journal of Orthopaedic Surgery 2007;15(1):41-5.

[15] Babu A, Rattan A, Ranjan P, et al. Are falls more common than road traffic accidents in pediatric trauma? Experience from a Level 1 trauma centre in New Delhi, India. Chinese Journal of Traumatology 2016;19(2):75-8.
[16] Singh O, Gupta S, Darokhan MA, et al. Epidemiology of pediatric musculoskeletal injuries and their pattern in a tertiary care center of North India. Indian Journal of Orthopaedics 2018;52(5):449-53.

[17] Cheng JC, Ng BK, Ying SY, et al. A 10-year study of the changes in the pattern and treatment of 6,493 fractures. Journal of Pediatric Orthopaedics 1999;19(3):344-50.

[18] Zionts LE, Zalavras CG, Gerhardt MB. Closed treatment of displaced diaphyseal both-bone forearm fractures in older children and adolescents. Journal of Pediatric Orthopaedics 2005;25(4):507-12.

[19] Teoh KH, Chee YH, Shortt N, et al. An age-and sexmatched comparative study on both-bone diaphyseal paediatric forearm fracture. Journal of Children's Orthopaedics 2009;3(5):367-73.

[20] Vaish A, Patwardhan S, Shyam A, et al. Surgical and functional outcomes of results of titanium elastic nailing system in paediatric diaphyseal fractures. Jour of Med Thesis 2016;4(1):26-30.

[21] Kubiak EN, Egol KA, Scher D, et al. Operative treatment of tibial fractures in children: an elastic stable intramedullary nail an improvement over external fixation? J Bone Joint Surg Am 2005;87(8):1761-8.

[22] Kumar P, Kisan D. Elastic nailing in diaphyseal fractures of femur in children. Journal of Orthopedics Traumatology and Rehabilitation 2018;10(2):107-11. 